\section{A Numerical Model of Permeabilized Skin With Local Transport Regions}

\author{
Nataša Pavšelj and Damijan Miklavčič*
}

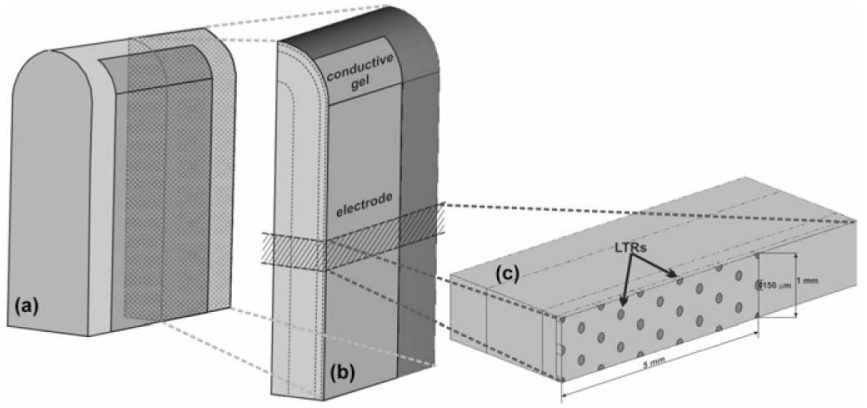

Fig. 1. (a) The geometry of the whole skin fold as it was during the in vivo experiments. (b) Only one-fourth of the skin fold was used to avoid numerica problems and save computer time. (c) Further, numerical model of skin with local transport regions was described as a slice of a quarter of the skin fold, using periodic boundary conditions. The thicknesses of the skin layers were: stratum corneum: $100 \mu \mathrm{m}$; viable epidermis: $250 \mu \mathrm{m}$; dermis: $1000 \mu \mathrm{m}$, subcutaneous layer: $650 \mu \mathrm{m}$. The diameter of local transport regions was $150 \mu \mathrm{m}$.

with a numerical model and compared the output of the model with our own in vivo experiments and previously published results of skin electroporation.

\section{METHODS}

\section{INTRODUCTION}

Cell membrane is, in general, impermeable for larger molecules; however, the application of electric pulses to cells, either in suspension or tissue, causes the electroporation of cell membrane, increasing its permeability and allowing larger molecules that otherwise cannot cross the membrane, such as drug molecules or DNA, to enter the cell [1]. Electroporation can also be used to create aqueous pathways across the skin's outermost layer, the stratum corneum, to enhance transdermal drug delivery or to enhance DNA transfection after intradermal and topical DNA delivery [2]-[5]. In the paper presented, the electropermeabilization process in skin is described theoretically, by means of numerical modeling, based on the in vivo experiments resulting from our previous research [6] and the data derived from the literature. When referring to its electric properties, skin is a very intricate tissue due to its highly inhomogeneous structure. Skin epidermis contains different layers, but the one that defines its electric properties the most is the highly resistive outermost layer, the stratum corneum, composed of dead, flat skin cells. Although very thin (typically around $20 \mu \mathrm{m}$ ), it contributes a great deal to the electric properties of the skin. Deeper skin layers all have much lower resistivity [7]-[9].

One phenomenon we can observe during in vivo experiments is an increase in tissue conductivity due to cell membrane permeabilization [10]-[12]. Further, it has been shown that the increase in the conductivity of the stratum corneum is not homogeneous throughout the electroporated area [13]-[17]. Molecular and ionic transport across the skin subjected to high-voltage pulses is highly localized in the socalled local transport regions (LTRs). We modeled these phenomena

Manuscript received June 5, 2007; revised January 8, 2008. This work was supported in part by the European Commission under the 5th Framework under Grant Cliniporator QLK-1999-00484, and in part by the Slovenian Research Agency. Asterisk indicates corresponding author.

N. Pavšelj is with the Faculty of Electrical Engineering, University of Ljubljana, SI-1000 Ljubljana, Slovenia (e-mail: natasa.pavselj@fe.uni-lj.si).

*D. Miklavčič is with the Faculty of Electrical Engineering, University of Ljubljana, SI-1000 Ljubljana, Slovenia (e-mail: damijan.miklavcic@fe. uni-lj.si).

Digital Object Identifier 10.1109/TBME.2008.919730
Electric field and electric current calculations were made by means of commercially available computer program COMSOL Multiphysics, version 3.3 (COMSOL, Los Angeles, CA, USA) based on finiteelement method. COMSOL Multiphysics is a powerful interactive environment for modeling and solving all kinds of scientific and engineering problems based on partial differential equations (PDEs). Models can be built through a flexible graphical user interface, or by script programming in the COMSOL Script language or in the MATLAB language.

\section{NUMERICAL MODEL}

Experiments show that the conductivity of a biological tissue changes during electroporation. We simulated this process with a numerical model of a skin fold, modeling tissue and electrode setups and pulse parameters used in the in vivo experiments made previously on rat skin [6]. Two parallel, stainless-steel plate electrodes of $4 \mathrm{~mm}$ distance were used to deliver electric pulses in vivo. Skin fold was formed and placed between the electrodes. The area of the electrodes in contact with skin was about $1 \mathrm{~cm} \times 1 \mathrm{~cm}$. A conductive gel was used in order to assure good contact between the skin and the electrodes, resulting in electric pulses being applied somewhat beyond the size of the electrodes. As we made no attempt on visualizing local transport regions during our in vivo experiments, we took the data on LTR size and density and their conductivity from the literature [13]-[17]. Four layers of skin were modeled: stratum corneum, viable epidermis, dermis, and the underlying layer of fat and connective tissue. The electrodes were modeled as a boundary condition. By taking advantage of the symmetry and applying proper boundary conditions, only one-fourth of the whole skin fold can be used by properly setting the boundary conditions on section planes. Further, only a slice of that part of the geometry can finally be modeled by setting periodic boundary conditions thus representing the skin fold with an array of local transport regions embedded in the stratum corneum. In this way, we avoided numerical problems due to the complexity of the model and computer memory limitations (see Fig. 1). 
TABLE I

INITIAL AND THE INCREASED SKIN LAYERS' CONDUCTIVITIES AND LTR PARAMETERS USED IN THE MODEL

\begin{tabular}{|c|c|c|}
\hline & $\begin{array}{l}\text { non- } \\
\text { permeabilized }\end{array}$ & $\begin{array}{l}\text { permeabilized / } \\
\text { LTR parameters }\end{array}$ \\
\hline subcut. tissue & $0.05 \mathrm{~S} / \mathrm{m}$ & $0.2 \mathrm{~S} / \mathrm{m}$ \\
\hline dermis, epidermis & $0.2 \mathrm{~S} / \mathrm{m}$ & $0.8 \mathrm{~S} / \mathrm{m}$ \\
\hline stratum corneum & $0.0005 \mathrm{~S} / \mathrm{m}$ & $\begin{array}{l}\text { LTRs: } \\
\text { - size: } 150 \mu \mathrm{m} \text { in diameter } \\
\text { - density: } 40 \mathrm{LTRs} / 0.1 \mathrm{~cm}^{2} \\
\text { - LTR surface: } 7 \% \text { of the stratum } \\
\text { corneum underneath the electrode } \\
\text { - LTR conductivity: } 1.25 \mathrm{~S} / \mathrm{m}\end{array}$ \\
\hline
\end{tabular}

When the electric field is applied to the skin fold, almost the entire voltage drop is on the outermost layer of the skin called the stratum corneum, which has the lowest conductivity. However, because of the occurrence of local transport regions in the stratum corneum, and thus the conductivity increase of the electropermeabilized stratum corneum, the electric field "penetrates" to the layers underneath [18]. Namely, when the electric field exceeds the predefined threshold, tissue conductivity increases. This change subsequently causes the change of the electric field distribution and of the corresponding electric current. Therefore, the final solution has to be reached iteratively. Also, once the conductivity was increased in a given area, it could not be changed back to its lower value in the following steps, even if the electric field strength should drop below the threshold due to changed conductivities. The electric conductivity values and their changes during electroporation were taken from the literature [7]-[12] and our own experiments, as well as the electric field electropermeabilization thresholds. The measurement of tissue electric properties can be complicated because of several factors, such as tissue inhomogeneity, anisotropy, the physiological state of the tissue, different animal species used, and electrode polarization. Also, the lack of standardization of the measurement technique leads to large discrepancies in the reported data on tissue electric properties. The conductivities used in our model were in the middle of the value ranges found in the literature. Further, data on virtually everything, except the conductivities before the electropermeabilization, are very scarce, sometimes nonexistent. Namely, the subject of tissue conductivity changes due to electroporation is still a rather unexplored field. However, with the model presented in this paper, we used the available data to explain the mechanism of the tissue electropermeabilization propagation beyond the initial conditions dictated by the tissue initial conductivities.

Exactly how tissue conductivities $(\sigma)$ change with electric field $(E)$ is another unknown of tissue electropermeabilization. Due to the nonuniformity of the cell size and shape in the tissue, not all the cells are permeabilized at the same time once the threshold electric field is reached. Therefore, we assumed a gradual increase of the conductivities with electric field. In our model, the conductivities were increased from their low to their high values in four steps between 600 and $1400 \mathrm{~V} / \mathrm{cm}$ of the electric field strength. The LTR parameters, the initial conductivities, and the increased conductivities of the permeabilized tissues for all skin layers modeled are summarized in Table I. Although the dermis and the viable epidermis were modeled as separate layers, the same conductivity was assigned to both, due to the lack of impedance data on different skin layers.

\section{RESUlTS AND DISCUSSION}

During our in vivo experiments [6], we used different pulse amplitudes. Five different voltages were used to permeabilize the skin:

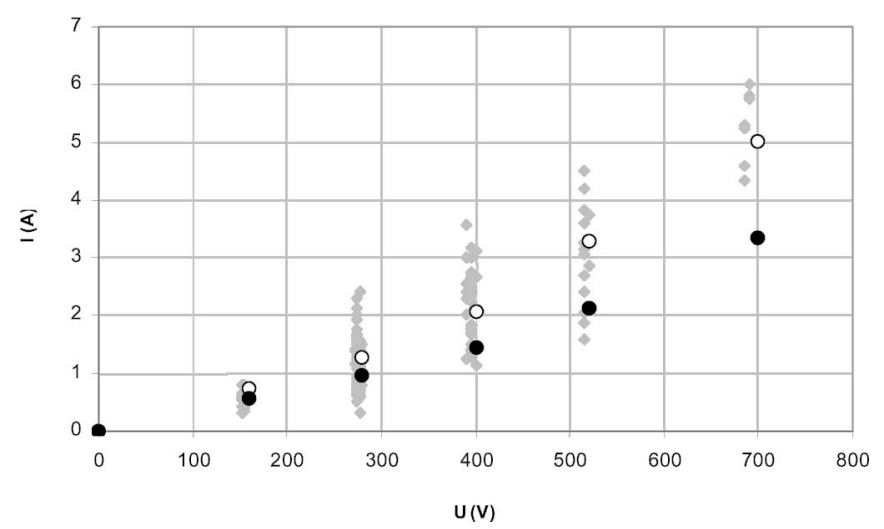

Fig. 2. Electric current obtained with the model (black circles), compared to the current measured during the pulse delivered in vivo (grey diamonds) and a previous model (open circles) [19] where stratum corneum was modeled as a homogeneous layer; with respect to the applied voltage.

$160,280,400,520$, and 700 V. During the pulse, the voltage between the electrodes and the electric current through the skin fold were measured. Our numerical model was also run five times, using the aforesaid voltages, and the electric currents given by the model were compared with our experimental data. Fig. 2 shows the currents of the model (black circles) compared to the currents measured in vivo during the pulse (grey diamonds). Also, we compared that to one of our previous models (open circles) [19], where stratum corneum was modeled as a homogeneous skin layer without local transport regions embedded in it. Its final conductivity during electroporation was also reached iteratively (the same procedure as used in the dermis, the viable epidermis, and the subcutaneous layer, described in Section III.

The electric currents obtained with our model are shifted toward somewhat lower values (1.3-1.5 times) also when compared to the previously published model. The reason for this can be attributed partially to different average conductivities of the permeabilized stratum corneum of both models. Namely, the average conductivity of the permeabilized part of the stratum corneum when LTRs are introduced is lower $(0.09 \mathrm{~S} / \mathrm{m}$ as opposed to $0.5 \mathrm{~S} / \mathrm{m}$ [19]). Also, the current-voltage dependence of the model with LTRs is more linear. In relation to this, it might be reasonable to construct different models for each applied voltage, with different numbers of local transport regions. Namely, as reported in the literature, the number of the LTRs increases with increasing voltage. Further, as the size, density, and the conductivity of local transport regions were taken from the literature, where different electroporation protocols were used than in our in vivo experiments, the parameters of the local transport regions formed in our experiments might have been different. Namely, trains of exponentially decaying pulses were used by the researchers reporting the visualization of the local transport regions, while only one square electroporative pulse was used in our experiments. Also, the tissue-electrode geometry was different, and more importantly, heat-separated human cadaver epidermis was used in their experiments, while our experiments were done in vivo on rat skin. Nevertheless, the results of the model are well in the range of in vivo experiments. We can conclude that the model presented describes skin electropermeabilization well by taking into account the increase of electrical conductivities of permeabilized skin layers and the presence of the local transport regions in the permeabilized stratum corneum.

Comparing the voltages needed for a successful electropermeabilization of the skin fold as suggested by the model, with voltages achieving efficient in vivo gene transfection (data not shown, some 
results published in [6]), a good agreement is observed. The voltage of $400 \mathrm{~V}$ between the plate electrodes resulted in a successful gene transfer in vivo. Also, the electric field distributions given by the model suggest that the electric field in the dermis and the viable epidermis is high enough for a successful electropermeabilization. The results also show that the inclusion of highly conductive areas in the stratum corneum leads to a nearly homogeneous permeabilization of the viable epidermis and the dermis, even though the conductivity increase in the stratum corneum is not homogeneous. In vivo experiments showed a lower expression when $160 \mathrm{~V}$ was used because the electric field in the viable epidermis and the dermis is still too low, as confirmed by the model. Similarly, the in vivo expression was lower when 520 or $700 \mathrm{~V}$ was used, due to lower cell viability caused by damaging high electric fields, which is again consistent with the results of the model, showing a very high electric field throughout the model at those voltages (data not shown).

Further, we compared our results (both in vivo and numerical) with already published findings on skin electropermeabilization. The voltage amplitudes resulting in a successful skin permeabilization during our in vivo experiments and the electric fields in the tissue given by the models agree well with the data we found in the literature [15].

\section{CONCLUSION}

Various in vivo experiments show a successful transdermal drug delivery or DNA delivery to the dermis and the viable epidermis by means of electroporation. As a result, electric conductivity of tissues subjected to electric pulses increases. Further, molecular and ionic transport across the skin was found to be highly localized in areas termed local transport regions (LTRs). We made a numerical model describing these phenomena, based on the finite-element method. The output of the model was compared to the currents and the voltages measured during in vivo experiments. Although the electric currents obtained with the model are somewhat lower (by factor 1.3-1.5), we can accept it as a good agreement. Namely, the data on LTR parameters were taken from the literature, where different experimental circumstances were used than in our own in vivo experiments. Further, comparing the voltages needed for a successful electropermeabilization of the skin fold as suggested by the model, with voltages achieving good in vivo gene transfection in our in vivo experiments, as well as those published by other authors, good agreement can be observed.

Some simplifications of numerical models were necessary due to their complexity. First, the stratum corneum was modeled six times thicker than in real skin to avoid large differences in layer thicknesses making the numerical calculation impossible. However, to make up for that, its conductivity was also set six times higher. Second, not enough skin fold volume was modeled at the base of the skin fold; thus, the natural electrical current flow was somewhat restrained. Third, with the periodic boundary conditions set on the boundaries of the skin fold slice with LTRs, an infinite array of slices was modeled. An error was made, as the electric field distribution around the borders of the electrodes differs from the distribution in the tissue situated well in the middle of the electrode. However, the error thus made was always below $10 \%$ (mostly of the order of $5 \%$ ), as estimated by numerical modeling and comparison of the currents in different areas of the LTR slice.

In the future, our numerical representation of skin electroporation will be improved by constructing different models for each applied voltage, with different numbers of local transport regions. Namely, as reported in the literature, the number of the LTRs increases with increasing voltage. Such models will better reflect the nonlinear current-voltage dependence of skin tissue during electroporation. Further, by coupling the electrical and the thermal phenomenon in the tissue, the thermal aspect of the LTR formation and expansion will also be investigated. Also, more in vivo experiments will be conducted with the aim of visualizing local transport regions as a result of skin electroporation. The validation of the improved numerical model with experimental results will thus be easier, as the geometry, the electroporative protocols, and the LTR parameters observed in vivo will directly be represented with numerical models. Nevertheless, with the model presented, we used the available data to describe the nonlinear process of skin electropermeabilization from the aspect of tissue conductivity changes and the presence of the local transport regions in the permeabilized stratum corneum. The observations derived from various in vivo experiments by different authors are thus confirmed theoretically. Such numerical models can successfully be used to predict the outcome of an experiment or a treatment when the geometry of the model closely represents the experimental setup and all the parameters, such as initial, permeabilized conductivities and their functional dependences are known. In their earlier stage, we have to be aware of their limitations; however, they still provide a valuable insight into the process.

\section{REFERENCES}

[1] L. M. Mir, "Therapeutic perspectives of in vivo cell electropermeabilization," Bioelectrochemistry, vol. 53, pp. 1-10, 2000.

[2] M. R. Prausnitz, "Reversible skin permeabilization for transdermal delivery of macromolecules," Crit. Rev. Ther. Drug Carrier Syst., vol. 14, no. 4, pp. 455-483, 1997.

[3] M. R. Prausnitz, "A practical assessment of transdermal drug delivery by skin electroporation," Adv. Drug Deliv. Rev., vol. 35, pp. 61-76, 1999.

[4] A.-R. Denet, R. Vanbever, and V. Préat, "Skin electroporation for transdermal and topical delivery," Adv. Drug Deliv. Rev., vol. 56, pp. 659-674, 2004.

[5] E. Neumann, S. Kakorin, and K. Toensing, "Fundamentals of electroporative delivery of drugs and genes," Biochim. Biophys. Acta, vol. 48, pp. 3-16, 1999.

[6] N. Pavšelj and V. Préat, "DNA electrotransfer into the skin using a combination of one high- and one low-voltage pulse," J. Control. Release, vol. 106, pp. 407-415, 2005.

[7] T. Yamamoto and Y. Yamamoto, "Electrical properties of the epiderma stratum corneum," Med. Biol. Eng., vol. 14, no. 2, pp. 151-158, 1976.

[8] T. Yamamoto and Y. Yamamoto, "Dielectric constant and resistivity of epidermal stratum corneum," Med. Biol. Eng., vol. 14, no. 5, pp. 494500, 1976.

[9] Y. A. Chizmadzhev, A. V. Indenbom, P. I. Kuzmin, S. V. Galichenko, J. C. Weaver, and R. O. Potts, "Electrical properties of skin at moderate voltages: Contribution of appendageal macropores," Biophys. J., vol. 74, pp. 843-856, 1998.

[10] M. R. Prausnitz, V. G. Bose, R. Langer, and J. C. Weaver, "Electroporation of mammalian skin: A mechanism to enhance transdermal drug delivery," Proc. Natl. Acad. Sci. U.S.A., vol. 90, pp. 10504-10508, 1993.

[11] U. Pliquett, R. Langer, and J. C. Weaver, "Changes in the passive electrical properties of human stratum corneum due to electroporation," Biochim. Biophys. Acta, vol. 1239, pp. 111-121, 1995.

[12] U. Pliquett and J. C. Weaver, "Electroporation of human skin: Simultaneous measurement of changes in the transport of two fluorescent molecules and in the passive electrical properties," Bioelectrochem. Bioenerg., vol. 39, pp. 1-12, 1996.

[13] U. F. Pliquett, R. Vanbever, V. Préat, and J. C. Weaver, "Local transport regions (LTRs) in human stratum corneum due to long and short 'high voltage' pulses," Bioelectrochem. Bioenerg., vol. 47, pp. 151-161, 1998.

[14] R. Vanbever, U. F. Pliquett, V. Préat, and J. C. Weaver, "Comparison of the effects of short, high-voltage and long, medium-voltage pulses on skin electrical and transport properties," J. Control. Release, vol. 69, pp. $35-47,1999$.

[15] J. C. Weaver, T. E. Vaughan, and Y. A. Chizmadzhev, "Theory of electrical creation of aqueous pathways across skin transport barriers," Adv. Drug Deliv. Rev., vol. 35, pp. 21-39, 1999. 
[16] U. Pliquett and C. Gusbeth, "Surface area involved in transdermal transport of charged species due to skin electroporation," Bioelectrochemistry, vol. 65 , pp. 27-32, 2004.

[17] U. Pliquett, S. Gallo, C. Gusbeth, E. Neumann, and S. W. Hui, "Local and transient perturbations in stratum corneum due to high voltage pulses: Contribution of local heating," Bioelectrochemistry, vol. 67, no. 1, pp. 37 46, 2005.
[18] N. Pavšelj, Z. Bregar, D. Cukjati, D. Batiuskaite, L. M. Mir, and D. Miklavčič, "The course of tissue permeabilization studied on a mathematical model of a subcutaneous tumor in small animals," IEEE Trans. Biomed. Eng., vol. 52, no. 8, pp. 1373-1381, Aug. 2005.

[19] N. Pavšelj, V. Préat, and D. Miklavčič, "A numerical model of skin electropermeabilization based on in vivo experiments," Ann. Biomed. Eng., vol. 35 , no. 12 , pp. 2138-2144, 2007. 